\title{
Interaction between horizontal and vertical fusional responses
}

\author{
DUANE K. BOMAN and ANDREW E. KERTESZ \\ Northwestern University, Evanston, Illinois
}

\begin{abstract}
Human fusional responses to combinations of horizontal and vertical disparity presentations were studied in order to determine how the horizontal and vertical fusional components are utilized during the response. Vertical fusional amplitudes decreased in the presence of large horizontal disparities. The reaction time to a vertical-step disparity increased when a horizontalstep disparity was simultaneously presented. Neither horizontal fusional amplitudes nor horizontal vergence responses to step or ramp disparity presentations were affected by the presence of vertical disparities. Thus, the results indicate a unilateral interaction between horizontal and vertical fusional components, one in which the fusibility of a compound disparity is affected by the order in which the disparity components are introduced.
\end{abstract}

Human fusional response to retinal image disparity contains two components: a motor component in the form of compensatory vengence eye movements and a nonmotor, or sensory, component whose magnitude is limited by the extent of Panum's fusional areas. Fusional responses have been studied using three types of stimulus disparity presentations: horizontal, vertical, and torsional. Thus, the fusional mechanism has been characterized in terms of horizontal, vertical, and cyclofusional responses. The responses elicited by these three types of disparity presentations were found to be quite different in speed, composition, and symmetry. Horizontal vergence eye movements are much faster than either vertical or cyclofusional movements (Perlmutter \& Kertesz, 1978; Rashbass \& Westheimer, 1961; Riggs \& Niehl, 1960; Sullivan \& Kertesz, 1978). The maximum amplitudes of horizontal motor and nonmotor fusional compensation are greater than the corresponding vertical amplitudes (Fender \& Julesz, 1967; Kertesz, 1981). Horizontal and cyclofusional vergence responses tend to be symmetric, while vertical vergence responses show asymmetries in their time course and monocular contribution to the overall motor response (Perlmutter \& Kertesz, 1978; Rashbass \& Westheimer, 1961; Sullivan \& Kertesz, 1978). Stimulus size, that is, the visual angle subtended by the stimulus, has different effects on the relative contributions of the motor and nonmotor components of horizontal, vertical, and cyclofusional responses (Kertesz, 1981; Sullivan \& Kertesz, 1978).

This work was supported in part by Research Grant EY1055 from the National Eye Institute. Please address all correspondence to: Andrew E. Kertesz, Biomedical Engineering Center, Northwestern University, 2145 Sheridan Road, Evanston, Illinois 60201.
Thus, differences among horizontal, vertical, and cyclofusional responses suggest that they may be mediated by separate mechanisms. All of the above responses, however, were observed in isolation, that is, disparities were presented along only one of the three orthogonal directions. But, under normal viewing conditions, the fusional mechanism is usually called upon to respond to composite disparities that may contain horizontal, vertical, and torsional components. How is the fusional response mediated under these circumstances? How are the horizontal, vertical, and cyclofusional response components utilized? Are the horizontal, vertical, and cyclofusional disparity components compensated independently or are there interactions among the three response components? Unfortunately, it is quite difficult to answer these questions, especially since the utilization of all three disparity components poses a special problem inasmuch as one could argue that torsional (or orientational) disparities might be compensated by combinations of horizontal and vertical nonmotor components. As unlikely as that outcome might be, especially in the light of experimental evidence to the contrary (Wright \& Kertesz, 1975), we decided to sidestep this issue by restricting our presentations to combinations of horizontal and vertical disparities. Therefore, the objective of these experiments was to examine the fusional response to stimuli containing combinations of horizontal and vertical disparities in order to determine how the quite different horizontal and vertical fusional components were utilized during the response.

\section{METHOD}

The stimulus was the stylized face shown in Figure 1. It was presented dichoptically on two CRTs with a short-persistence 


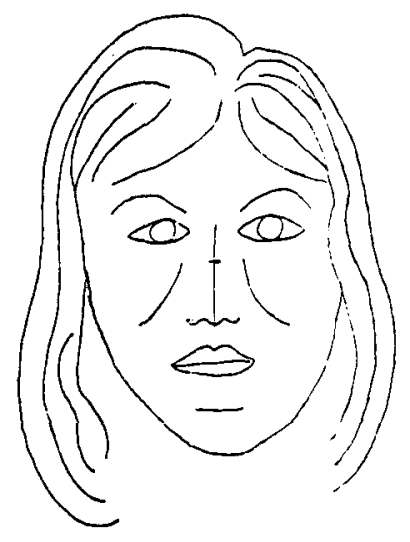

Figure 1. Stylized face used as the experimental stimulus.

P15 phosphor, was viewed at a distance of $51.4 \mathrm{~cm}$, and subtended $8.78 \mathrm{deg}$ vertically and $6.56 \mathrm{deg}$ horizontally. Stimulus disparities could be introduced in increments of $14 \mathrm{sec}$ of arc in both the horizontal and vertical directions. Only divergent horizontal and right-hyper vertical disparities were used. A right-hyper vertical disparity is said to exist between the monocular stimulus images if the stimulus presented to the right eye is displaced in an upward direction with respect to the stimulus seen by the left eye.

In the last two experiments, two SRI double Purkinje image eyetrackers were used to monitor the horizontal and vertical positions of each eye. The eye-movement signals were sampled at a rate of $10 \mathrm{~Hz}$ in the second experiment and at $100 \mathrm{~Hz}$ in the third experiment. Disjunctive (vergence) eye-movement records were computed by subtracting the right-eye positions from the corresponding left-eye positions. A detailed description of the eyetrackers was given by Cornsweet and Crane (1973). The resolution of the system was evaluated by using an artificial eye and was found to be $1 \mathrm{~min}$ of arc for both horizontal and vertical movements. The baseline drift for the eyetrackers over a period of $10 \mathrm{~min}$ was also within $1 \mathrm{~min}$ of arc. The eyetrackers were found to be linear within $2 \%$ for 2 degree movements in any direction.

Four subjects were used during these experiments. Each had a normal fusional range and a small horizontal phoria of less than 3 prism diopters, which was compensated during the experiments and required a slight spherical correction to achieve $20 / 20$ acuity. One subject (J.J.B.) also had a 1-prism-diopter vertical phoria that was compensated. The subject was seated in a dark room in order to eliminate conflicting spatial cues from his field of view. His head was held rigidly by a tight-fitting dental impression plate and a foreheadrest.

\section{RESULTS}

\section{Experiment 1}

The effect of orthogonal disparities on fusional amplitudes was studied in this experiment. Fusional amplitudes in both the horizontal and vertical directions were measured under three conditions: (A) in the presence of zero orthogonal disparity; (B) in the presence of an orthogonal disparity that was large but easily fusible; and $(C)$ in the presence of an orthogonal disparity that was slightly below the subject's fusional amplitude.

Each subject was given a training period during which his horizontal and vertical fusional amplitudes were measured in the presence of zero orthogonal disparity. The subject was able to increase or decrease the disparity between the stimuli in steps of $2.5 \mathrm{~min}$ of arc in the horizontal direction or $1.5 \mathrm{~min}$ of arc in the vertical direction. He was asked to find the largest disparity at which the stimuli were perceived as single.

When a subject's fusional amplitudes became repeatable, horizontal and vertical disparity values that corresponded to the three conditions stated above were chosen. These disparities will be referred to as initial disparities. Each subject's fusional amplitudes were then measured 30 times under each of the six conditions, that is, three for horizontal and three for vertical.

The initial disparity was introduced in the form of a ramp at the rate of $3.9 \mathrm{~min}$ of arc/sec in the vertical direction or $7.7 \mathrm{~min}$ of arc/sec in the horizontal direction. A tone indicated when the initial disparity value had been reached. The subject was then instructed to find his fusional amplitude in the orthogonal direction.

The average horizontal fusional amplitudes that were measured in the presence of zero or nonzero vertical disparities and their standard deviations are shown in Table 1. The initial vertical disparities are also expressed as a percentage of the subject's vertical fusional amplitude evaluated in the presence of zero horizontal disparity. A one-way analysis of variance showed no significant changes in any of the subjects' horizontal fusional amplitudes in the presence of vertical disparities. Even vertical disparities that approximated $98 \%$ of a subject's vertical fusional amplitude had no significant effect on his horizontal fusional amplitude.

The average vertical fusional amplitudes that were measured in the presence of horizontal disparities and their standard deviations are shown in Table 2. Again, the initial disparities are also expressed as a percentage of the subject's horizontal fusional amplitude in the presence of zero vertical disparity. A one-way analysis of variance showed significant changes in the vertical fusional amplitudes of Subjects D.K.B. $(p<.05)$ and D.R.H. $(p<.01)$ in the presence of horizontal disparities. A test comparing each of the vertical fusional amplitudes measured in the presence of nonzero horizontal disparities with each of those measured in the presence of zero horizontal disparity showed a significant decline in D.K.B.'s and D.R.H.'s vertical fusional amplitudes under Condition $C(p<.01)$, but not under Condition B. This test also showed a significant decline in Subject A.R.P.'s vertical fusional amplitude under Condition $C(p<.01)$. The fact that this change was not detected by the analysis of variance for Subject A.R.P. is probably due to the large standard deviation that this subject had under Condition B. Table 2 shows that the decline in the vertical fusional 
Table 1

Experiment 1: Horizontal Fusional Amplitudes in Minutes of Arc

\begin{tabular}{|c|c|c|c|c|c|c|c|c|c|c|c|}
\hline \multirow[b]{3}{*}{ Subject } & \multicolumn{3}{|c|}{ Condition 1} & \multicolumn{4}{|c|}{ Condition 2} & \multicolumn{4}{|c|}{ Condition 3} \\
\hline & \multirow[t]{2}{*}{ IVD } & \multicolumn{2}{|c|}{ FA } & \multicolumn{2}{|c|}{ IVD } & \multicolumn{2}{|c|}{ FA } & \multicolumn{2}{|c|}{ IVD } & \multicolumn{2}{|c|}{ FA } \\
\hline & & Mean & SD & $\mathrm{HA}$ & PVA & Mean & SD & $\mathrm{HA}$ & PVA & Mean & SD \\
\hline D.K.B. & 0 & 517 & 14 & 80 & 71 & 497 & 8 & 110 & 98 & 502 & 10 \\
\hline A.R.P. & 0 & 485 & 21 & 90 & 64 & 474 & 8 & 110 & 79 & 495 & 31 \\
\hline J.J.B. & 0 & 578 & 73 & 100 & 52 & 612 & 62 & 180 & 93 & 519 & 63 \\
\hline D.R.H. & 0 & 617 & 12 & 120 & 57 & 627 & 68 & 150 & 71 & 602 & 47 \\
\hline
\end{tabular}

Note-IVD = initial vertical disparity; FA = fusional amplitude; $S D=$ standard deviation $; H A=$ horizontal fusional amplitude; $P V A=$ percentage of vertical fusional amplitude.

Table 2

Experiment 1: Vertical Fusional Amplitudes in Minutes of Arc

\begin{tabular}{|c|c|c|c|c|c|c|c|c|c|c|c|}
\hline \multirow[b]{3}{*}{ Subject } & \multicolumn{3}{|c|}{ Condition 1} & \multicolumn{4}{|c|}{ Condition 2} & \multicolumn{4}{|c|}{ Condition 3} \\
\hline & \multirow[t]{2}{*}{ IHD } & \multicolumn{2}{|c|}{ FA } & \multicolumn{2}{|c|}{ IHD } & \multicolumn{2}{|c|}{ FA } & \multicolumn{2}{|c|}{ IHD } & \multicolumn{2}{|c|}{ FA } \\
\hline & & Mean & SD & VA & PHA & Mean & SD & VA & PHA & Mean & SD \\
\hline D.K.B. & 0 & 111 & 8 & 414 & 80 & 117 & 6 & 484 & 94 & 97 & 3 \\
\hline A.R.P. & 0 & 140 & 10 & 434 & 89 & 132 & 19 & 464 & 96 & 123 & 17 \\
\hline J.J.B. & 0 & 192 & 39 & 434 & 74 & 189 & 23 & 474 & 81 & 198 & 15 \\
\hline D.R.H. & 0 & 211 & 12 & 454 & 74 & 197 & 33 & 552 & 89 & 153 & 31 \\
\hline
\end{tabular}

Note-IHD = initial horizontal disparity; FA = fusional amplitude; $S D=$ standard deviation; $V A=$ vertical fusional amplitude; $P H A=$ percentage of horizontal fusional amplitude.

amplitudes of three of the four subjects occurred only in the presence of horizontal disparities that approximated $90 \%$ of that subject's horizontal fusional amplitude.

Figure 2 illustrates the unilateral interaction between horizontal and vertical fusional compensation for Subject D.K.B. Although the presence or absence of a 110-min vertical disparity did not affect the magnitude of the horizontal fusional amplitude, the presence of a 484-min horizontal divergent disparity significantly reduced the vertical fusional amplitude from 111 to $97 \mathrm{~min}$. If the disparity components are introduced sequentially, then the order of introduction may determine whether the overall disparity will be fusible or not. For example, if the disparity consists of 110-min vertical and 484-min horizontal

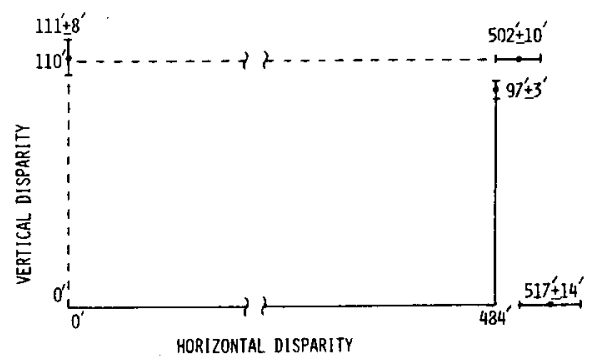

Figure 2. Fusional amplitudes (with standard deviations) of Subject D.K.B. in response to horizontal, vertical, or combinations of horizontal and vertical stimulus disparities. components, it will be fusible if the vertical disparity is introduced before the horizontal disparity (dashed path in Figure 2). If, on the other hand, the horizontal disparity component is introduced first, then the overall disparity will not be fusible (solid path in Figure 2).

\section{Experiment 2}

In Experiment 1, the effect of orthogonal disparities on fusional amplitudes were studied. In this experiment, the effect of orthogonal disparities on the motor and nonmotor components of the fusional responses were evaluated by the measurement of fusional vergence responses to horizontal and vertical ramp disparity presentations. Two disparity values were chosen in each of the two directions for each subject. One was chosen to be as large as the subject could repeatedly fuse, whereas the other was well below this value. The subject's vergence eye movements were monitored in response to the introduction of each of the horizontal and vertical disparities in the presence of either zero or one of the two nonzero orthogonal disparities. Each presentation was replicated 20 times.

Each trial began with zero disparity between the stimuli. Disparities were introduced at the rate of $11.6 \mathrm{~min}$ of arc/sec in the horizontal direction and $2.9 \mathrm{~min}$ of $\mathrm{arc} / \mathrm{sec}$ in the vertical direction. The entire disparity in one direction was introduced before any orthogonal disparity was. Three subjects were 
used in this experiment, and the following disparities were presented: 320 and 480 min horizontal and 80 and $120 \mathrm{~min}$ vertical for Subject D.R.H.; 320 and $440 \mathrm{~min}$ horizontal and 60 and $90 \mathrm{~min}$ vertical for Subjects A.R.P. and D.K.B.

In this experiment, $81 \%$ to $97 \%$ of the horizontal disparity was compensated by horizontal vergence eye movements, while the magnitude of vertical fusional motor compensation ranged from $75 \%$ to $121 \%$ of the vertical disparity. This left a small, but significant, nonmotor component in many cases. A one-way analysis of variance was employed to detect changes in the level of motor compensation to a specific disparity in the presence of different orthogonal disparities. None of the subjects showed significant changes in their levels of motor compensation to horizontal disparities in the presence of vertical disparities. Only Subject A.R.P. showed a significant change in his vertical motor compensation in the presence of horizontal disparities. His vertical vergence movements significantly increased in response to both the $60-\mathrm{min}(\mathrm{p}<.01)$ and $90-\mathrm{min}$ $(p<.05)$ vertical disparities when either of the nonzero horizontal disparities were also present. For example, his percentage of motor compensation in response to a 60 -min vertical disparity changed from $75 \%$ in the presence of zero horizontal disparity to $93 \%$ in the presence of a 320-min horizontal disparity and to $88 \%$ in the presence of a 440 -min horizontal disparity.

\section{Experiment 3}

In the first two experiments, fusional responses to slowly increasing disparities were tested. In this experiment, variations in reaction times and levels of motor compensation were studied in response to combinations of step disparities. Fusional vergence responses were monitored during the introduction of step disparities in both the horizontal and vertical directions. Five conditions were presented in which: (A) the horizontal and vertical steps occurred simultaneously; (B) the vertical step occurred $5 \mathrm{sec}$ after the horizontal step; (C) the horizontal step occurred $10 \mathrm{sec}$ after the vertical step; (D) the vertical step occurred while the eyes were still responding to the horizontal step; and (E) the horizontal step occurred while the eyes were still responding to the vertical step.

All four subjects participated in this experiment. Eye-movement recordings began $5 \mathrm{sec}$ prior to the first disparity step and ended $10 \mathrm{sec}$ after the second disparity step. Each of the five conditions was replicated 20 times.

Reaction times and the levels of horizontal and vertical motor compensation were evaluated. The horizontal reaction times ranged from 131 to $180 \mathrm{msec}$, and the vertical reaction times ranged from 135 to $370 \mathrm{msec}$. The percentages of motor compensation

ranged from $70 \%$ to $102 \%$ in the horizontal direction, and from $55 \%$ to $122 \%$ in the vertical direction. A one-way analysis of variance was employed to detect changes in either the reaction times or the levels of motor compensation. There were no significant changes in the levels of motor compensation. There were also no significant changes in the horizontal reaction times. Significant changes were found in the vertical reaction times for Subjects D.K.B. ( $p<.01)$ and D.R.H. $(p<.01)$. Each subject's vertical reaction time under Condition $C$, which corresponds to presentation of a vertical-step disparity alone, was compared with his vertical reaction times under the other four conditions. It was revealed that the vertical reaction times under Condition $\mathrm{A}$ were significantly longer than those under Condition $C$ for both Subject D.K.B. and Subject D.R.H. $(p<.01)$. None of the other conditions had a significant effect on the subject's vertical reaction times. Subject D.K.B.'s vertical reaction time increased from $230 \mathrm{msec}$ under Condition $\mathrm{C}$ to $300 \mathrm{msec}$ under Condition A. Subject D.R.H. showed a corresponding increase from 155 to $228 \mathrm{msec}$.

Figure 3 shows typical responses of Subject D.K.B. under Conditions $\mathrm{A}$ and $\mathrm{C}$. The horizontal vergence responses are similar under the two conditions. There is a slight convergence in response to the verticalstep-disparity presentation under Condition $C$, but the eyes return to their previous positions within a second. The vertical vergence responses also show

A
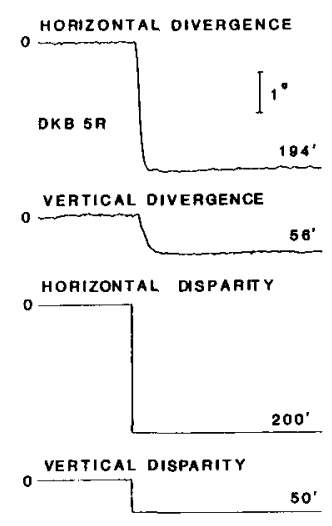

Figure 3. Disjunctive (vergence) eye-movement responses of Subject D.K.B. to combined horizontal- and vertical-step-disparity presentations. The disjunctive eye-movement responses were calculated by subtracting the left- from the right-eye positions in the horizontal and vertical directions $(D=L-R)$. The top two traces depict the horizontal- and vertical-vergence eye movements. The bottom two traces depict the horizontal and vertical stimulus disparities. The stimulus step disparities were divergent horizontal and right-hyper vertical. (A) An average of five responses by D.K.B. to the simultaneous presentation of 200 -min horizontal and 50-min vertical-step disparities. (B) An average of five responses by D.K.B. to a 50 -min vertical-step disparity that was followed, after $10 \mathrm{sec}$, by a 200 -min horizontal-step disparity. 
little change other than the longer reaction time under Condition A. Again, there is a slight vertical vergence in response to the horizontal-step presentation under Condition $C$, with the eyes returning to their previous vertical positions within a second.

\section{DISCUSSION}

Studies of a complex system usually begin with the examination of each of the response components in isolation, and only in subsequent stages are inquiries made as to how these components are utilized to produce a more complex response. Thus, it was found that horizontal and vertical vergence responses are quite different and are probably mediated by separate mechanisms. The differences are not due to the utilization of different combinations of extraocular muscles, because horizontal and vertical version responses are similar (Goodwin \& Fender, 1973). The objective of this study was to examine how the fusional mechanism combines its horizontal and vertical components to respond to a complex disparity. Our results show that horizontal fusional responses were unaffected by either the presence of vertical disparities or by simultaneously ongoing vertical fusional responses. Vertical fusional responses, on the other hand, were affected by both the presence of horizontal disparities and by horizontal fusional responses. This suggests a unilateral interaction from horizontal to vertical disparity processing. The nature of this interaction is such that the fusional compensation of a complex disparity containing horizontal and vertical components is a function of the order in which the disparity components are introduced.

It is not surprising to observe interactions between horizontal and vertical eye movements. Complex bilateral interactions have been reported between horizontal and vertical saccadic systems (Feinstein \& Williams, 1972a, 1972b), as well as between horizontal and vertical version responses (Goodwin \& Fender, 1973). But it is surprising to find unilateral interactions, because, to the best of our knowledge, interactions of this type have never been reported for the fusional mechanism or for the oculomotor system.

Horizontal fusional responses are necessary for the sequential bifoveation of objects located at different distances from the observer. Horizontal disparity detection is also utilized in stereopsis. These considerations place strong demands on the fusional mechanism and result in a highly developed horizontal fusional response. It may be for this reason that the more developed horizontal fusional response is unaffected by the presence of vertical disparities.

It is well known that fusional amplitudes are functions of stimulus parameters. Fender and Julesz (1967) found that the contribution of the nonmotor components, the extent of Panum's fusional areas, is also a function of the recent history of stimulation. Our results indicate that, due to a unilateral interaction between horizontal and vertical fusional responses, the processing of composite disparities also depends on the recent history of stimulation, in that the order of presentation of the disparity components becomes significant.

We have also observed that the simultaneous presentation of vertical- and horizontal-step disparities results in a significant increase of vertical reaction time. A simple sequential processing scheme may explain this result, but it would not account for the decrease in vertical fusional amplitude when it is measured in the presence of a previously applied horizontal disparity. One might argue that the increase in vertical reaction time is due to the additional need for interhemispheric communication imposed by the simultaneous introduction of a rather large horizontal disparity. Purely vertical stimulus disparities may be detected without interhemispheric communication, and it is only when they are combined with a horizontal disparity whose magnitude exceeds the central region of bihemispheric representation that the detection of vertical disparities adjacent to the vertical midline of the visual field will require interhemispheric communication. However, the detection of large horizontal disparities about the midline routinely require interhemispheric communication, yet horizontal reaction times are not significantly different from vertical reaction times. Thus, it seems that neither the sequential processing scheme nor the need for interhemispheric communication can adequately explain the increased vertical reaction time.

In summary, if separate mechanisms mediate horizontal and vertical fusional responses, they cannot be considered independent but interactive, in which case the processing of composite disparities is accomplished by a unilateral interaction from the horizontal to the vertical disparity processing mechanism.

\section{REFERENCES}

Cornsweet, T. N., \& Crane, H. D. Accurate two dimensional eyetrackers using first and fourth images. Journal of the Optical Society of America, 1973, 62, 921-928.

Feinstein, R., \& Williams, W. J. Interactions of the horizontal and vertical human oculomotor systems: The saccadic systems. Vision Research, 1972, 12, 33-44. (a)

Feinstein, R., \& Williams, W. J. Interaction of the horizontal and vertical human oculomotor systems: The vertical smooth pursuit and horizontal saccadic systems. Vision Research, 1972, 12, 45-52. (b)

Fender, D. H., \& Julesz, B. Extension of Panum's fusional areas in binocular stabilized vision. Journal of the Optical Society of America, 1967, 57, 819-830.

Goopwin, A. W., \& Fender, D. H. The interaction between horizontal and vertical eye-rotation in tracking tasks. Vision Research, 1973, 13, 1701-1712. 
Kertesz, A. E. Effect of stimulus size on fusion and vergence. Journal of the Optical Society of America, 1981, 71, 289-293.

Perlmutter, A., \& Kertesz, A. E. Measurement of human vertical fusional response. Vision Research, 1978, 18, 219-223.

Rashbass, C., \& Westheimer, G. Disjunctive eye movements. Journal of Physiology, 1961, 159, 339-360.

RigGs, L. A., \& NiEht, E. W. Eye movements recorded during convergence and divergence. Journal of the Optical Society of America, 1960, 50, 913-920.
Sullivan, M. J., \& Kertesz, A. E. Binocular coordination of torsional eye movements in cyclofusional response. Vision Research, 1978, 18, 943-949.

Wright, J. C., \& Kertesz, A. E. The role of positional and orientational disparity cues in human fusional response. Vision Research, 1975, 15, 427-430.

(Manuscript received July 9, 1982;

revision accepted for publication February 17, 1983.) 